\title{
Development of Flexible Film Mold for Nanoimprinting Process
}

\author{
Yasuo Suto*, Misato Yamanaka, Yoshiho Urata, Hiroko Yamada, \\ Yukihiro Miyazawa and Takahide Mizawa \\ Soken Chemical \& Engineering Co., Ltd., 1-13-1, Hirosehigashi, Sayama-shi, \\ Saitama-ken, Japan
}

Keywords: nanoimprint, film mold, roll-to-roll

\section{Introduction}

Nanoimprint technology is a type of surface microfabrication technology that has been derived from the well known emboss technique in the optical disk production [1]. Conventional nanoimprinting molds, which are made of silicon or quartz, are not always suitable for practical mass-production owing to its high cost and low throughput [2]. Generally, replica molds are used to overcome those problems. As a replication material, polydimethylsiloxane (PDMS) is well used. However, PDMS doesn't always have high durability against transcription materials, because transcription materials tend to be penetrated into PDMS. Furthermore, large area nanoimprinting molds cannot be easily obtained through conventional mold fabrication process despite they are needed to achieve the mass production of imprinted product.

In this study, to achieve the mass production, we developed replica film mold made of specific resin. Durability and transfer properties against transcription material of both the film mold and common PDMS were evaluated. Furthermore, to extend applications of film mold, micro-nano mixed structure was fabricated in film mold as we reported [3]. Finally, the film mold was applied to the roll-to-roll (R2R) UV nanoimprinting process, because high throughput is essential to achieve the mass production.

\section{Experimental}

Our film mold was fabricated by UV nanoimprinting from our original resin A on PET substrate as reported [4]. PDMS mold was fabricated by thermal process from PDMS precursor solution. As a master mold, micro pillar patterned nickel mold, whose pattern height was $1.7 \mu \mathrm{m}$, was used. Continuous UV nanoimpritng was conducted with the film mold or PDMS mold. As a transcription material, OrmoComp ${ }^{\circledR}$ (Micro Resist Technology $\mathrm{GmbH}$ ), a kind of hybrid polymer, was used.

As one of applications of micro-nano mixed structure, decorative film mold which is composed of nanopillar pattern and line and space pattern was fabricated based on previous studies [3]. In addition, enlargement of the decorative film mold was processed through appropriate step and repeat process we developed [5]. Outer dimension of the decorative film mold was $300 \mathrm{~mm} \times 850 \mathrm{~mm}$. The enlarged film mold was attached on the roll cylinder and continuous UV R2R nanoimprinting was conducted on the condition as follows: the line speed was 1.4

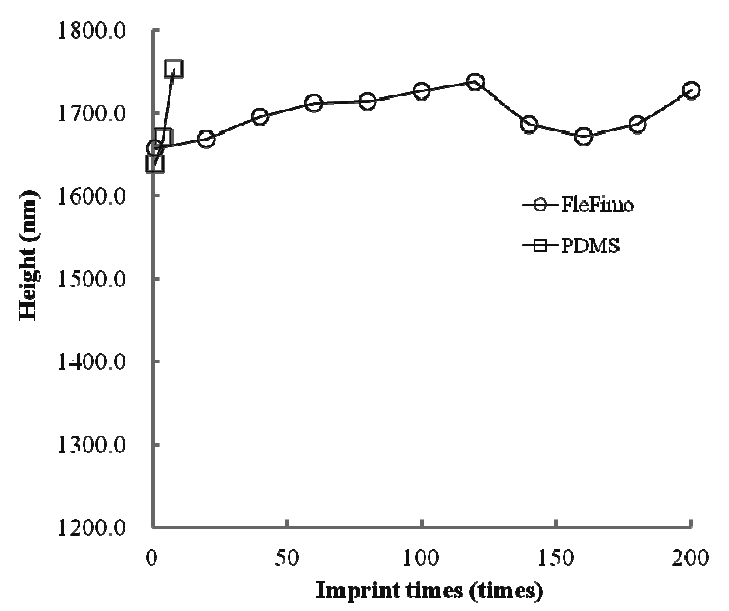

Fig. 1. Durability and transfer property of film mold and PDMS mold against OrmoComp ${ }^{\circledR}$. 
$\mathrm{m} / \mathrm{min}$ and accumulated energy density of UV irradiation was around $2,000 \mathrm{~mJ} / \mathrm{cm}^{2}$.

Each fine structure was measured by field emission scanning electron microscopy (FE-SEM, Hitachi S-4800) or conventional atomic force microscopy (AFM, Hitachi L-trace).

\section{Results and discussion}

The film mold exhibited several superior properties such as excellent mold releasability, high transmittance in the UV-Vis range and appropriate mechanical properties.

Fig. 1 shows durability and transfer property of the film mold and PDMS mold against OrmoComp ${ }^{\circledR}$. Apparent stuck OrmoComp ${ }^{\circledR}$ was observed on PDMS after 8 times imprint. In addition, pattern height of the imprinted product was gradually changed with the increase of imprint times. PDMS is known to swell during imprinting process, because transcription materials penetrate into PDMS. On the other hand, when our film mold was used, apparent pattern deformation of imprinted products was not observed even after 200 times imprint in a row. These results indicate that our film mold had high durability and high transfer property against hybrid polymer compared to PDMS mold.

Not only just replication, different patterns were mixed on one film mold to produce highly functional films. Fig. 2a shows the appearance of the enlarged decorative film mold. Fig. $2 b$ shows the SEM image of the decorative film mold. Both of line and space pattern and nanopillar pattern were observed. They were successfully integrated on one film mold based on the design using conventional master mold.

The decorative film mold was applied to UV $\mathrm{R} 2 \mathrm{R}$ nanoimpriting process to demonstrate that our film mold is useful for mass-production. It was conducted as shown in Fig. 3a. The imprinted product, which has apparent structural color based on fine structure, was obtained from decorative film mold as shown Fig. $3 b$. Furthermore, Fig. 3c shows the SEM image of imprinted product. The inverted structure of the decorative film mold was apparently observed. Finally, $170 \mathrm{~m}$ decorative film was successfully obtained through the R2R process. Obtained imprinted product had high transcription accuracy without remarkable defects.

These results strongly indicate that our film mold will be adaptable for producing a large amount of various functional films.

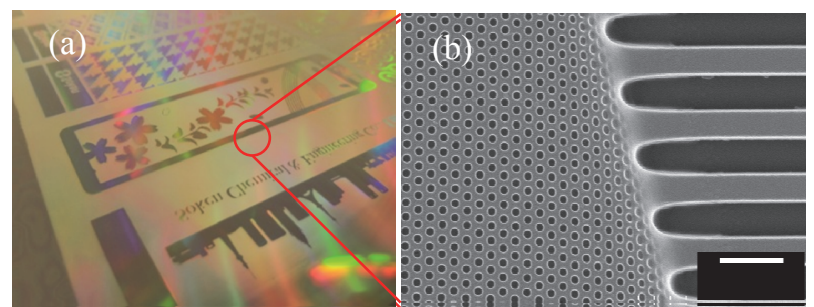

Fig. 2. (a) Appearance and (b) SEM image of fabricated micro-nano mixed structure mold.

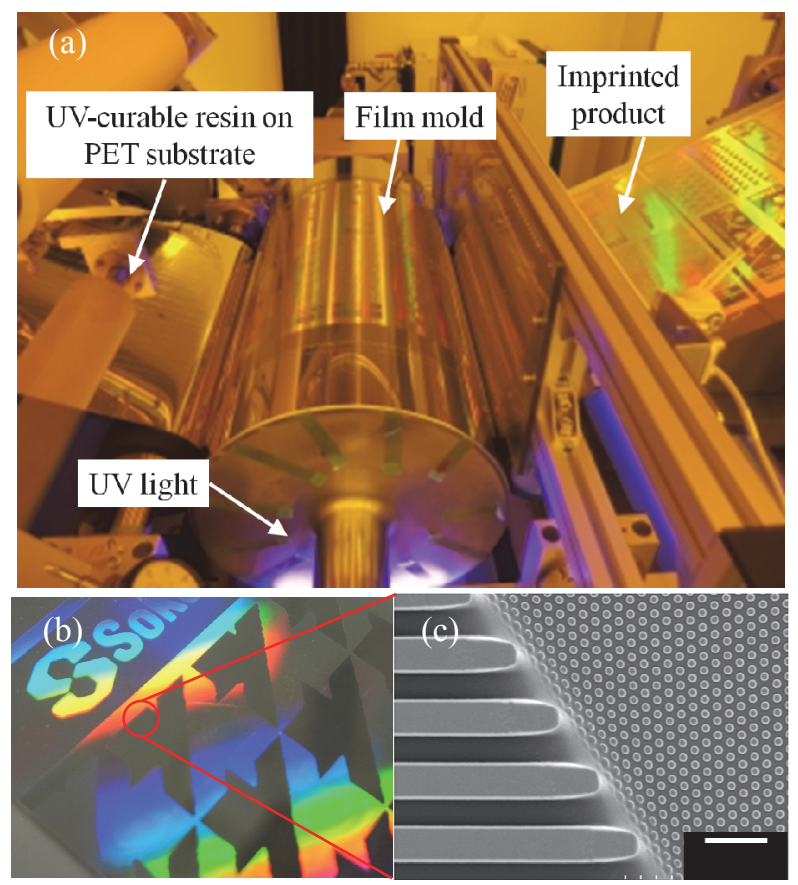

Fig. 3. (a) Production of imprinted product by R2R UV nanoimprinting process, (b) appearance and (c) SEM image of imprinted product of micro-nano mixed structure mold.

\section{Conclusion}

Our film mold had higher durability compared to PDMS mold. In addition, to provide highly functional films, specific film mold which has nano-micro mixed structure was successfully obtained. The film mold continuously produced the imprinted product through $\mathrm{R} 2 \mathrm{R}$ process. The film mold can be applied for achieving mass production of imprinted products.

\section{References}

1. S. Y. Chou, P. R. Krauss and P. J. Renstrom, $J$. Vac. Sci. Technol. B, 14 (1996) 4129.

2. F. Reuther, J. Photopolym. Sci. Technol., 18 (2005) 525.

3. Y. Suto, Y. Miyazawa, Y. Mizukami and T. Mizawa, Abstract of Nanoimprint \& Nanoprint Technology, (2014) 24A-6-3.

4. Y. Suto, T. Mizawa, Y. Mizukami and T. Takahashi, J. Photopolym. Sci. Technol., 26 (2013) 125-128.

5. Y. Suto, Y. Miyazawa and T. Mizawa, submitted to J. Photopolym. Sci. Technol., (2016). 